cerebellar syndrome. These patients usually have cerebellar cortical atrophy. ${ }^{2}$

Autosomal dominant cerebellar ataxia resembles idiopathic cerebellar ataxia in many respects. Like idiopathic cerebellar ataxia, autosomal dominant cerebellar ataxia is a late onset disorder that may present as a pure cerebellar disorder or in combination with non-cerebellar symptoms. Neuropathological findings in idiopathic cerebellar ataxia and autosomal dominan cerebellar ataxia are often indistinguishable. These phenomenological similarities have prompted the hypothesis that at least some cases of idiopathic cerebellar ataxia arise from spontaneous mutation of a gene responsible for autosomal dominant cerebellar ataxia, from non-paternity, or falsely appear sporadic due to incomplete pedigree data. ${ }^{14}$ The recent discovery of an expanded trinucleotide repeat mutation in the SCA1 region in American families with autosomal dominant cerebellar ataxia offers the opportunity to search for this mutation in isolated patients with idiopathic cerebellar ataxia by the polymerase chain reaction. 5

We have analysed DNA from 61 patients with idiopathic cerebellar ataxia with a mean age of 53.6 (SD 12.8) years and a mean disease duration of 9.2 (SD 47) years. All patients satisfied the following diagnostic criteria: (a) progressive, otherwise unexplained ataxia, and (b) family history without evidence of heredity or consanguinity of parents. All diagnoses were made after exclusion of possible symptomatic causes (alcoholism, other toxic causes, malignancy, hypothyroidism, vitamin deficiency, inflammatory, metabolic, or vascular causes). Twenty five patients had a pure cerebellar syndrome (idiopathic cerebellar ataxia-C), whereas 36 had additional non-cerebellar symptoms (idiopathic cerebellar ataxia-P). Of these, 17 fulfilled the clinical criteria for probable multiple system atrophy. Polymerase chain reaction analysis was performed, as described by Orr et al. ${ }^{5}$ For comparison, DNA from 144 patients with atosomal dominant cerebellar ataxia was analysed. As a positive control, DNA from families with autosomal dominant cerebellar ataxia from Yakut tribes from Siberia was included, in which the SCAl expansion has been demonstrated and sequenced.

A diagnosis of SCA1 heterozygocity was made in five out of 19 German families with autosomal dominant cerebellar ataxia for which the family history was medically documented. This corresponds to a prevalence of SCA1 in German families with autosomal dominant cerebellar ataxia of about $25 \%$. By contrast, the SCA1 expansion was found in none of the 61 cases of idiopathic cerebellar ataxia.

The present results argue against the hypothesis that a relevant number of cases of idiopathic cerebellar ataxia are due to a trinucleotide expansion at the SCAl locus. The importance of our failure to show a mutation of the SCAl gene in patients with idiopathic cerebellar ataxia is underlined by the finding that the SCA1 mutation is present in a considerable proportion of German families with autosomal dominan cerebellar ataxia. Our data do not exclude the possibility that some cases of idiopathic cerebellar ataxia are due to spontaneous mutations at other gene loci associated with autosomal dominant cerebellar ataxia. Linkage studies provide evidence that there are at least two other gene loci (SCA2 and SCA3) for autosomal dominant cerebellar ataxia. ${ }^{67}$ The hypothesis that idiopathic cerebellar ataxia is due to a mutation at one of these loci cannot be tested at present because SCA2 and SCA3 genes are not identified. It is unlikely that a recessive gene defect accounts for many cases of idiopathic cerebellar ataxia because recessive inheritance of a late onset ataxic disorder has been documented only very rarely. It therefore seems likely that most cases of idiopathic cerebellar ataxia are non-inherited. This hypothesis is supported by a recent study reporting discordance for idiopathic cerebellar ataxia in monzygous triplets. ${ }^{4}$ In addition, detailed clinical, neuroradiological, and neuropathological investigations show subtle differences between idiopathic cerebellar ataxia and autosomal dominan cerebellar ataxia. Thus age of onset is more variable and tends to be lower in autosomal dominant cerebellar ataxia. Additional noncerebellar features of idiopathic cerebellar ataxia are often parkinsonism and autonomic failure, whereas ophthalmoplegia, pyramidal signs, dystonia, and amyotrophy are more common in autosomal dominant cerebellar ataxia. Pathological and neuroradiological studies show that there is frequent spinal involvement in autosomal dominant cerebellar ataxia but not in idiopathic cerebellar ataxia. ${ }^{2}$ Finally, postmortem studies have shown the presence of oligodendroglial intracytoplasmatic inclusions in the brains of patients with sporadic olivopontocerebellar atrophy, but not in the brains of patients with dominantly inherited olivopontocerebellar atrophy. ${ }^{8}$

T KLOCKGETHER K BURK J DICHGANS

Department of Neurology University of Tübingen, Hoppe-Seyler-Strasse 3,
(1) D-72076 Tübingen, Germany Department of Neurology, University of Lübeck, Ratzeburger Allee 160 D-23562 Lübeck, Germany G AUBURGER

Department of Neurology,

University of Dïsseldorf,

D-40225 Düsseldorf, Germany

Correspondence to: $\mathrm{Dr} T$ Klockgether, Tübingen, Hoppe-Seyler-Strasse 3, D-72076 Tübingen, Germany.

1 Harding AE. "Idiopathic" late onset cerebellar ataxia. A clinical and genetic study of 36 ataxia. A clinical and genetic study

2 Klockgether T, Wüllner U, Dichgans J, et al. Clinical and imaging correlations in inherited ataxias. Adv Neurol 1993;61:77-96.

3 Quinn N. Multiple system atrophy-the nature of the beast. $\mathcal{F}$ Neurol Neurosurg Psychiatry 1989;52 (special supplement) 78-89.

4 Harding AE, Sura T, Tippett P, Boughey AM, Patten JP. Discordance for late-onset degenerative ataxia in monozygous triplets. F Neurol 1991;238:242-3.

5 Orr HT, Chung MY, Banfi S, et al. Expansion of an unstable trinucleotide repeat in spinocerebellar ataxia type 1. Nature Genetics 1993;4:221-6.

6 Gispert S, Twells R, Orozco G, et al. Chromosomal assignment of the second (cuban) locus for autosomal dominant cerebellar ataxia (SCA2) to human chromosome 12q23-24:1. Nature Genetics 1993;4:295-9.

7 Takiyama $Y$, Nishizawa $M$, Tanaka $H$, et al. The gene for Machado-Joseph disease maps to gene for Machado-Joseph disease maps to human chrom

8 Papp MI, Kahn JE, Lantos PL. Glial cytoplasmatic inclusions in the CNS of patients with multiple system atrophy (striatonigral degeneration, olivopontocerebellar atrophy, and Shy-Drager syndrome). $\mathcal{F}$ Neurol Sci 1989;94:79-100

\section{Life threatening epilepsy in a child}

Epilepsy is known to cause a variety of arrhythmias that sometimes lead to sudden death..$^{1-4}$ These arrhythmias are evoked by abnormal autonomic activity to the heart. ${ }^{235}$ We encountered a patient with epilepsy who developed torsades de pointes. Torsades de pointes-an ECG manifestation in which wide QRS like ventricular tachycardia continuously appears with periodically changing polarity-sometimes leads to ventricular fibrillation, which can cause sudden death. ${ }^{6}$ To our knowledge, this is the first reported incidence of epilepsy that caused torsades de pointes.

A 7 year old girl, who had been investigated for two attacks of epilepsy during the previous six months, was admitted to our institution after respiratory arrest. There was no relevant family history. Her pupils were dilated and there was no light reflex. An ECG showed ventricular fibrillation. After intubation, two attempts at cardioversion with $50 \mathrm{~J}$ restored normal sinus rhythm. She was then admitted to the intensive care unit and maintained under controlled ventilation. The ECG taken on admission to the intensive care unit was normal, with no prolongation of the QT interval $\left(\mathrm{QT}_{\mathrm{c}}=0.44 \mathrm{~s}\right)$. About two hours later, a generalised convulsion developed, followed by repeated premature ventricular contractions that immediately changed into torsades de pointes (figure). Xylocaine was ineffective, but diazepam finally terminated both the convulsion and the torsades de pointes. Serum electrolyte concentrations (K $4.5 \mathrm{mmol} / \mathrm{l}, \mathrm{Ca} 9.0 \mathrm{mmol} / \mathrm{l}, \mathrm{Mg} 2 \cdot 1$ $\mathrm{mg} / \mathrm{dl})$, and $\mathrm{PaO}_{2}(280 \mathrm{mmHg})$ were all normal at this time. Thiamylal sodium (3 $\mathrm{mg} / \mathrm{kg} / \mathrm{h}$ ) was given for the next three days. During this period the ECG was also normal, with no prolongation of the QT interval. The patient became able to talk two days after barbiturate withdrawal. Myocarditis and cardiomyopathy were ruled out on the basis of echocardiographic findings, and she was negative for all the viral antibodies tested. An EEG obtained in the interictal period was normal. Cranial CT also showed no abnormalities. Thus the arrhythmia was diagnosed as torsades de pointes evoked by generalised seizures. After having been placed on oral anticonvulsant treatment, the patient was transferred to another institution 11 days later without any neurological deficits.

The two points of interest in this case were the induction of torsades de pointes by generalised seizures and the termination of both the seizures and the arrhythmia by diazepam. In this patient, the epileptic focus was not determined, so the attacks were classified as generalised seizures on the basis of their clinical features. As the development of torsades de pointes was preceded by a generalised seizure, the arrhythmia was assumed to be attributable to the seizure. There have been no similar reports. As both conditions developed almost simultaneously, xylocaine was initially given to terminate her torsades de pointes without prolongation of the QT interval, but proved to be ineffective. There was not sufficient time to apply other agents or overdrive pacing. Both conditions were terminated, however, by the diazepam that was subsequently given to control the seizure. Diazepam specifically acts on the limbic system. Considering that arrhythmias secondary to seizures are mediated by a limbic-forebrain- 


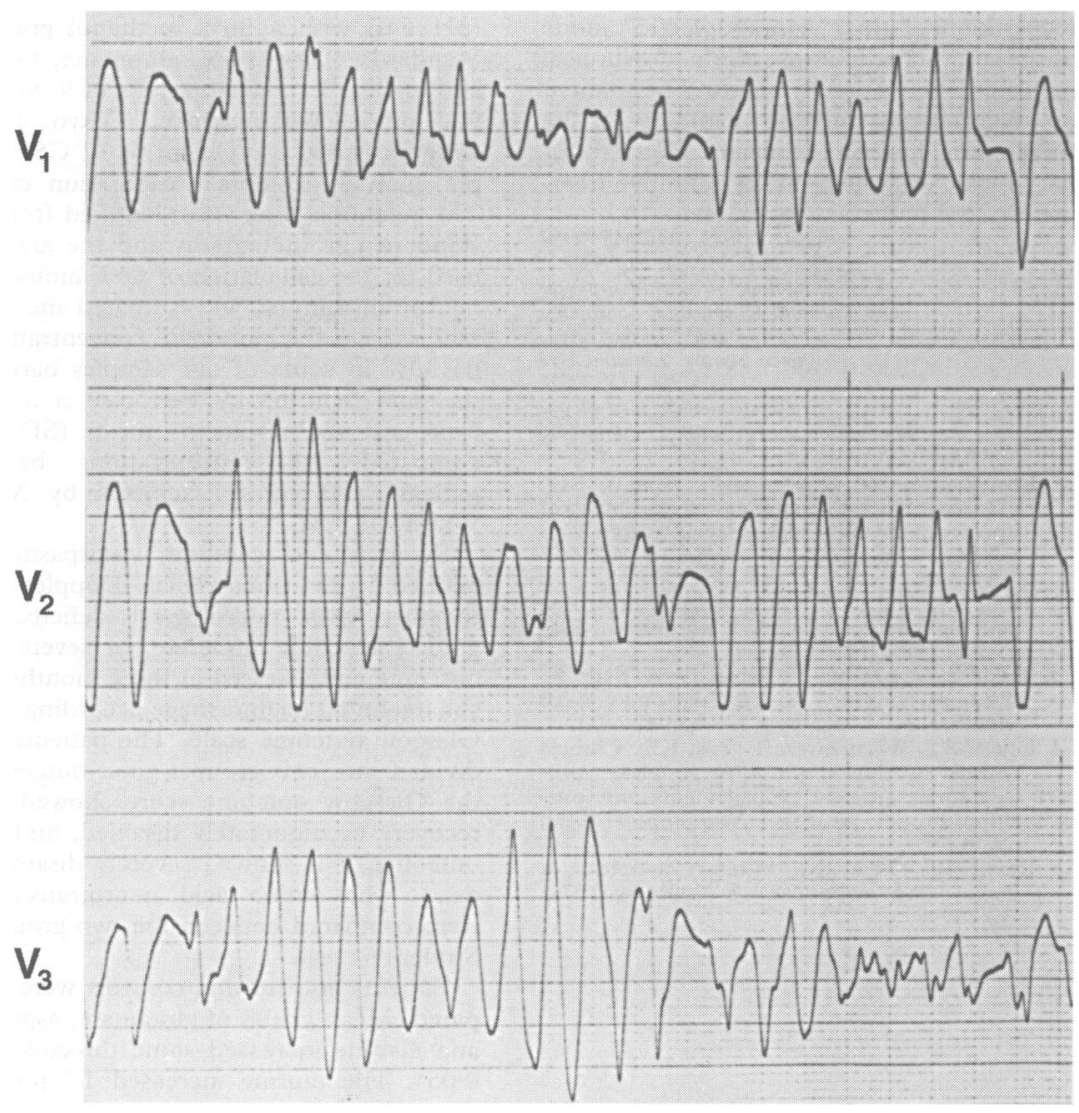

Electrocardiogram showing torsades de pointes. An electromyogram was not obtained because the generalised convulsion ceased.

midbrain circuit, ${ }^{5}$ the present findings are interesting and may assist in the treatment of similar patients.

$$
\begin{array}{r}
\text { TOSHIHIKO KUROIWA } \\
\text { HIROSHI MORITA } \\
\text { HARUSHI TANABE } \\
\text { TOMIO OHTA } \\
\text { Osaka Mishima, } \\
\text { Critical Care Medical Center and } \\
\text { Department of Neurosurgery, } \\
\text { Osaka Medical College, } \\
\text { Osaka, fapan }
\end{array}
$$

Correspondence to: $\mathrm{Dr}$ Toshihiko Kuroiwa, Osaka Mishima Critical Care Medical Center, 11-1 Minami Akutagawa Cho, Takatsuki City, 11-1 Minami A

Osaka 569, Japan.
1 Dasheiff RM, Dickinson LVJ. Sudden unex- pected death of epileptic patient due to cardiac arrhythmia after seizure. Arch Neurol diac arrhythmia

2 Leestma JE, Kalelkar MB, Teas SS, et al. Sudden unexpected death associated with seizures: analysis of 66 cases. Epilepsia 1984; 25:84-8.

3 Jay GW, Leestma JE. Sudden death in epilepsy: a comprehensive review of the literature and proposed mechanism. Acta Neurol Scand 1981;63(suppl 82):1-66.

4 Terrence CF, Wisotzkey HM, Perper JA Unexpected, unexplained death in epileptic patients. Neurology 1975;25:594-8.

5 Gilchrist JM. Arrhythmogenic seizures. Diagnosis by simultaneous EEG/ECG Diagnosis by simultaneous EEG/EC
recording. Neurology 1985;35:1503-6.

6 Dessertenne F. La tachycardie ventriculaire a deux foyers opposes variables. Arch Mal deux foyers opposes
Coeur 1966;59:263-72.

\section{Atypical presentation of vascular events in pituitary tumours: "non-apoplectic" pituitary apoplexy}

Sudden disabling headache, visual deterioration, or impaired conscious level (sometimes in the context of a known pituitary adenoma under medical treatment) is the classical presentation accompanying acute haemorrhage or infarction of the pituitary gland. ${ }^{1}$ We have recently managed three cases in which radiologically and pathologically confirmed vascular events in pituitary tumours presented with ophthalmoplegia in the absence of changes in either visual fields or acuity and in which headache was a minor symptom. This atypical mode of presentation may confound accurate diagnosis and delay appropriate treatment. Surgery for this condition is associated with good examination was normal. Brain MRI showed an intrasellar mass compressing the left cavernous sinus and displacing the optic chiasm (figure). Magnetic resonance angiography performed at the same time excluded an aneurysm. At transphenoidal hypophysectomy infarcted pituitary tissue that was under some tension was evacuated; there was a rim of apparently normal tissue evident at the periphery of the lesion. After the operation there was a rapid improvement in the diplopia, which has subsequently completely resolved. Histopathological examination showed infarcted pituitary tissue; there was insufficient viable tumour to allow for immunohistochemistry.

Case 2, a 52 year old man, developed a sudden drooping of his left eyelid accompanied by a mild headache. There had been no alteration in his vision and there was no history of endocrine disorder. Examination showed ptosis and proptosis of the right eye with complete ophthalmoplegia and pupillary involvement. Fundoscopy was normal. Corrected visual acuity was $6 / 9$ on the right and $6 / 5$ on the left; the visual fields were full. Magnetic resonance imaging showed a sellar mass of mixed high signal with suprasellar extension to the optic chiasm, which was clearly compressing the right cavernous sinus and displacing the right internal carotid artery. The appearances were typical of tumour infarction with haemorrhage. At transphenoidal hypophysectomy a large quantity of overtly necrotic pituitary tumour was removed. Histology confirmed necrosis and haemorrhage. Immediately after operation the ocular movements began to improve. At the time of discharge the fourth and sixth nerve palsies had fully recovered but the third nerve palsy remained evident.

Case 3, a 78 year old woman, originally presented with ophthalmoplegia due to a pituitary adenoma. This had almost completely resolved 18 months after transphenoidal hypophysectomy when she developed diplopia of a few days duration accompanied by drooping of the left eyelid. The patient complained of a mild discomfort behind the left eye but no headache. She was alert and lucid. A pupil sparing left third nerve palsy and also a partial left sixth

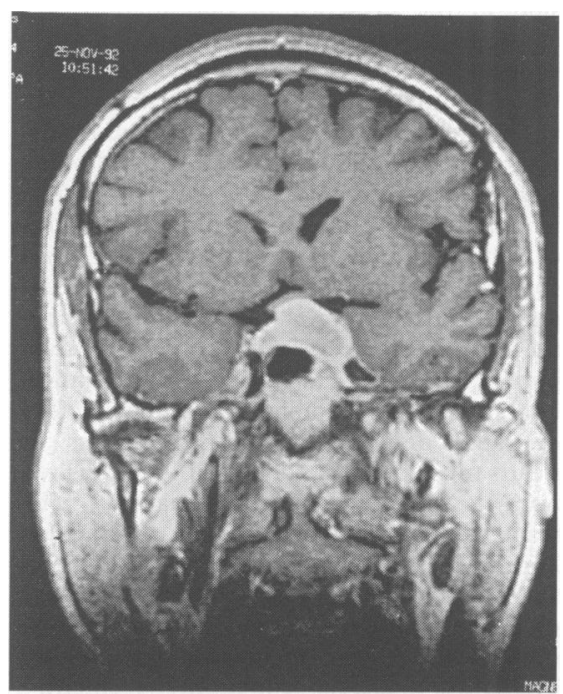

Postcontrast coronal MRI (case 1) showing a homogeneous sellar mass with ring enhancement. There is pronounced compression of the left cavernous sinus. 